\title{
Association between abdominal aortic plaque and coronary artery disease
}

This article was published in the following Dove Press journal:

Clinical Interventions in Aging

19 May 2016

Number of times this article has been viewed

\author{
Wei $\mathrm{Li}^{1,2, *}$ \\ Songyuan Luo 2 ,* \\ Jianfang Luo ${ }^{2}$ \\ Yuan Liu \\ Wenhui Huang ${ }^{2}$ \\ Jiyan Chen ${ }^{2}$
}

'Department of Cardiology, Southern Medical University, Guangzhou, People's Republic of China; ${ }^{2}$ Department of Cardiology, Guangdong Provincial Cardiovascular Institute, Guangdong General Hospital, Guangdong Academy of Medical Sciences, Guangzhou, People's Republic of China

*These authors contributed equally to this work
Correspondence: Jianfang Luo Department of Cardiology, Guangdong Provincial Cardiovascular Institute, Guangdong General Hospital, Guangdong Academy of Medical Sciences, No 106, Zhongshan 2nd Road, Guangzhou, Guangdong 510080, People's Republic of China

Tel +86 2083827812

Email henryjfl@tom.com
Objective: Currently, the association between abdominal aortic plaques and coronary artery disease (CAD) has not yet been clarified clearly. The purpose of this study was to determine the prevalence of abdominal aortic plaques by ultrasound imaging and to explore its association with $\mathrm{CAD}$ in patients undergoing coronary angiography.

Methods: Between October 2014 and June 2015, a prospective study was conducted in the Department of Cardiology at Guangdong General Hospital, Guangzhou, People's Republic of China. Ultrasound scanning of the abdominal aortas was performed in 1,667 consecutive patients undergoing coronary angiography. Clinical characteristics and coronary profile were collected from the patients.

Results: Of the 1,667 study patients (male, 68.9\%; mean age, $63 \pm 11$ years) undergoing coronary angiography, 1,268 had CAD. Compared with 399 patients without CAD, 1,268 patients with $\mathrm{CAD}$ had higher prevalence of abdominal aortic plaques $(37.3 \%$ vs $17 \%, P<0.001)$. In multivariate analysis, abdominal aortic plaques served as independent factors associated with the presence of $\mathrm{CAD}$ (odds ratio $=2.08 ; 95 \%$ confidence interval $=1.50-2.90 ; P<0.001$ ). Of the 1,268 patients with CAD, the prevalence of abdominal aortic plaques was $27.0 \%(98 / 363)$ in patients with one-vessel disease, $35.0 \%$ (107/306) in patients with two-vessel disease, and $44.7 \%(268 / 599)$ in patients with three-vessel disease. Stepwise increases in the prevalence of abdominal aortic plaque was found depending on the number of stenotic coronary vessels $(P<0.001 ; P$-value for trend $<0.001)$. In an ordinal logistic regression model, abdominal aortic plaques served as independent factors associated with the severity of $\mathrm{CAD}$ according to the number of stenotic coronary vessels $(P<0.001)$.

Conclusion: The prevalence of abdominal aortic plaques was higher in patients with CAD than in those without CAD. Abdominal aortic plaque was an independent factor associated with the presence and severity of CAD.

Keywords: abdominal aortic plaque, coronary artery disease, ultrasound imaging, atherosclerosis

\section{Introduction}

Atherosclerotic disease is a main cause of severe cardiovascular events, such as myocardial infarction and stroke. As the prevalence of atherosclerosis increases globally due to aging population, identifying atherosclerotic plaques will be of utmost importance for early diagnosis and intervention, which will substantially decrease the health care burden. ${ }^{1}$

Evidence is available indicating that subclinical atherosclerosis in carotid artery is associated with the presence and severity of coronary artery disease (CAD) and with an increased 10-year risk of cardiovascular events. ${ }^{2-5}$ The association between thoracic aortic plaques and $\mathrm{CAD}$ has been often reported by using transesophageal 
echocardiography. ${ }^{6,7}$ Furthermore, researchers found that the extent of thoracic aortic atheroma burden was independently associated with increased long-term mortality in patients following cardiothoracic surgery. ${ }^{8}$ Although autopsy studies reported plaques in the abdominal aorta to be severe in patients with cardiac catastrophe, ${ }^{9}$ there are limited studies showing the association between CAD and abdominal aortic plaques. Only a small number of observational studies reported that the prevalence and extents of plaques in abdominal aortas were greater in patients with CAD than in those without CAD by using cardiovascular magnetic resonance (CMR). But they reported that plaques in abdominal aorta were not independent factors for CAD. ${ }^{9-11}$ Besides, due to lack of large-scale data, the results of these studies may not be convictive. Although CMR was a useful tool for evaluating atherosclerotic plaques in abdominal aortas, ${ }^{12}$ it is neither cost efficient nor suitable for population-based studies.

The 2014 ESC guidelines recommend that population screening for abdominal aortic aneurysm (AAA) should be performed by abdominal aorta ultrasound in all men $>65$ years of age. ${ }^{13}$ Abdominal aorta ultrasound has become a useful tool to not only screen AAA but also evaluate atherosclerotic plaques, which is noninvasive, easy to assess, cost efficient, and could be routinely used in population-based study. ${ }^{1}$ However, the significance of abdominal aortic plaques detection by ultrasound imaging is not yet definitive.

The association between CAD and abdominal aortic plaques has not yet been clarified clearly. The purpose of the present study was to determine the prevalence of abdominal aortic plaques by ultrasound imaging and to explore its association with CAD in patients undergoing coronary angiography.

\section{Subjects and methods Study patients}

Between October 2014 and June 2015, a prospective study was conducted in the Department of Cardiology at Guangdong General Hospital, Guangzhou, People's Republic of China. The study was approved by the ethics committees of Guangdong General Hospital. Abdominal aortic ultrasound is a routine examination in patients undergoing coronary angiography in our hospital. As this was an observational study, written informed consent was not required according to the policy of the ethics committees of Guangdong General Hospital. Ultrasound scanning of the abdominal aortas was performed in 1,667 consecutive patients undergoing coronary angiography for suspected or known CAD, or prior to valve surgery. Excluded were patients with aortic diseases, congenital heart disease, infectious diseases, autoimmune diseases, or neoplasma. The clinical characteristics of the patients and their coronary profile were collected prospectively.

\section{Coronary angiography}

Coronary angiography was performed using a standard technique. Obstructive $\mathrm{CAD}$ was defined as the existence of astenosis $>50 \%$ of the lumen diameter of at least one major coronary vessel. The severity of the disease referred to the number of identified stenosed vessels (lumen diameter $<50 \%$ ) [one-, two-, or three-vessel disease]) and was evaluated by two experienced cardiologists, who were blinded to the ultrasound evaluation of the abdominal aortas. ${ }^{3}$

\section{Assessment of abdominal aortic atherosclerosis}

Atherosclerosis of the abdominal aortas was assessed by ultrasound scanning with a ultrasound device equipped with a Philips iE 33 imager with a 3.5/7 MHz multiplane probe. All studies were recorded and were reviewed offline by two independent senior echocardiographers who were blinded to patient demographics as well as to coronary profile. A third expert was advised in case of disagreement, and the majority view was adopted.

Abdominal aortic intimal morphology was graded by a modification of the classification proposed by Pitsavos et al: $;{ }^{14}$ thus, atherosclerotic plaque characteristics and maximum width were graded as follows: grade I, normal intima; grade II, increased intimal echo density without lumen irregularity; grade III, increased intimal echo density with single or multiple well-defined atheromatous plaque $\leq 3 \mathrm{~mm}$; and grade IV, atheroma $>3 \mathrm{~mm}$ or mobile or ulcerated plaque. Each patient was characterized on the basis of the most severe identified lesion. Grades III and IV were considered to represent abdominal aortic plaque. ${ }^{15}$

\section{Evaluation of risk factor variables}

The risk factor variables evaluated in the present study included age, sex, smoking, hypertension, diabetes mellitus, chronic kidney disease, triglyceride, total cholesterol, low density lipoprotein-cholesterol (LDL-C), and high density lipoprotein-cholesterol (HDL-C).

Hypertension was defined as present if there was any history of high blood pressure or if the blood pressure measured twice in the hospital exceeded $140 \mathrm{mmHg}$ (systolic) or $90 \mathrm{mmHg}$ (diastolic) or in case of current use of antihypertensive medications.

Diabetes mellitus was defined as present if the patient had a history of diabetes or if the fasting plasma glucose exceeded 7.0 or $11.1 \mathrm{mmol} / \mathrm{L} 2$ hours after a meal or in case of current use of diabetic medications. 
Chronic kidney disease was defined as present if the estimated glomerular filtration rate (eGFR) was less than $60 \mathrm{~mL} / \mathrm{min} / 1.73 \mathrm{~m}^{2}$. The eGFR (expressed as $\mathrm{mL} / \mathrm{min} / 1.73 \mathrm{~m}^{2}$ ) was computed using a prediction formula derived from the Modification of Diet in Renal Disease study: ${ }^{16}$ eGFR $=186 \times$ $\left(\right.$ serum creatinine $\left.{ }^{-1.154}\right) \times\left(\right.$ age $\left.^{-0.203}\right) \times 0.742$ (if female).

Blood samples measured by standard laboratory methods were taken in a fasting state on the morning of the day when angiography was performed.

\section{Statistics}

Quantitative data are presented as mean \pm standard deviation (SD) and qualitative data are presented as frequencies. Differences between two groups were evaluated by the unpaired $t$-test for parametric variables and by the chi-square test for categorical variables. A multiple logistic regression analysis was used to elucidate the associations between abdominal aortic plaque and CAD. An ordinal logistic regression analysis was used to elucidate the associations between abdominal aortic plaque and the severity of CAD. A $P$-value of $<0.05$ was considered statistically significant. All the statistical analyses were made with the SPSS for Windows software, version 20.0 (IBM Corporation, Armonk, NY, USA).

\section{Results}

\section{Prevalence of abdominal aortic plaques in patients with CAD}

Of the 1,667 study patients (male, $68.9 \%$; mean age, $63 \pm 11$ years) undergoing coronary angiography, 1,268 had CAD ( $>50 \%$ stenosis). Compared with 399 patients without CAD, 1,268 patients with CAD had higher prevalence of abdominal aortic plaque (37.3\% vs $17 \% ; P<0.001)$. Other clinical characteristics in patients with and without $\mathrm{CAD}$ are displayed in Table 1.

\section{Factors associated with the presence of CAD}

To identify any independent factor associated with the presence of $\mathrm{CAD}$, clinical variables (age, sex, smoking, hypertension, diabetes mellitus, chronic kidney disease, triglyceride, total cholesterol, LDL cholesterol, HDL cholesterol, abdominal aortic plaque) were entered into a multivariate logistic regression model. In multivariate analysis, male sex (odds ratio [OR] $=2.45 ; 95 \%$ confidence interval $[\mathrm{CI}]=1.76-3.41 ; P<0.001)$, diabetes mellitus $(\mathrm{OR}=1.54 ; 95 \% \mathrm{CI}=1.09-2.17 ; P=0.014)$, and abdominal aortic plaque $(\mathrm{OR}=2.08 ; 95 \% \mathrm{CI}=1.50-2.90 ; P<0.001)$ were independent factors associated with the presence of CAD (Table 2).
Table I Clinical characteristics in patients with and without CAD

\begin{tabular}{|c|c|c|c|}
\hline Characteristics & $\begin{array}{l}\text { CAD (+) } \\
(\mathrm{N}=1,268)\end{array}$ & $\begin{array}{l}\text { CAD (-) } \\
(\mathrm{N}=399)\end{array}$ & $P$-value \\
\hline Age (years) & $63.57 \pm 10.60$ & $61.24 \pm 0.68$ & $<0.001$ \\
\hline Sex (male) & $947(74.7)$ & $201(50.4)$ & $<0.001$ \\
\hline Smoking & $464(36.6)$ & $92(23.1)$ & $<0.001$ \\
\hline Hypertension & $778(6 \mid .4)$ & $187(46.9)$ & $<0.001$ \\
\hline Diabetes mellitus & $365(28.8)$ & $68(17)$ & $<0.001$ \\
\hline CKD & $233(18.4)$ & $38(9.5)$ & $<0.001$ \\
\hline Triglyceride & $1.63 \pm 1.25$ & $1.57 \pm 1.24$ & $0.4 \mathrm{II}$ \\
\hline Total cholesterol & $4.56 \pm 1.16$ & $4.38 \pm 1.24$ & 0.009 \\
\hline LDL-C (mmol/L) & $2.62 \pm 0.99$ & $2.57 \pm 0.99$ & 0.445 \\
\hline HDL-C (mmol/L) & $1.04 \pm 0.27$ & I. $17 \pm 0.34$ & $<0.001$ \\
\hline AAP & $473(37.3)$ & $68(17)$ & $<0.001$ \\
\hline
\end{tabular}

Note: Data are presented as the mean \pm SD or the number (\%) of patients. Abbreviations: CKD, chronic kidney disease; LDL-C, low density lipoproteincholesterol; HDL-C, high density lipoprotein-cholesterol; AAP, abdominal aortic plaque; $C A D$, coronary artery disease; $S D$, standard deviation.

\section{Prevalence of abdominal aortic plaques according to the coronary profile}

Of the 1,268 patients with CAD, 363 had one-vessel disease, 306 had two-vessel disease, and 599 had three-vessel disease. The prevalence of abdominal aortic plaques was $27.0 \%(98 / 363)$ in patients with one-vessel disease, $35.0 \%$ (107/306) in patients with two-vessel disease, and $44.7 \%$ (268/599) in patients with three-vessel disease. As shown in Figure 1, stepwise increases in the prevalence of abdominal aortic plaque were found to depend on the number of stenotic coronary vessels $(P<0.001 ; P$-value for trend $<0.001)$.

\section{Factors associated with the severity of CAD}

To identify any independent factor associated with the severity of CAD according to the number of stenotic coronary vessels, clinical variables (age, sex, smoking, hypertension, diabetes mellitus, chronic kidney disease, triglyceride, total cholesterol, LDL-cholesterol, HDL-cholesterol, and

Table 2 Factors associated with the presence of CAD (multiple logistic regression analysis of the I,667 study patients)

\begin{tabular}{llll}
\hline Variables & OR & $\mathbf{9 5 \% ~ C l}$ & P-value \\
\hline Sex (male) & 2.45 & $1.76-3.41$ & $<0.001$ \\
Diabetes mellitus & 1.54 & $1.09-2.17$ & 0.014 \\
AAP & 2.08 & $1.50-2.90$ & $<0.001$ \\
\hline
\end{tabular}

Notes: The dependent variables indicate the presence of CAD. The factors analyzed included age, sex, smoking, hypertension, diabetes mellitus, chronic kidney disease, triglyceride, total cholesterol, LDL-cholesterol, HDL-cholesterol, and abdominal aortic plaque.

Abbreviations: OR, odds ratio; $\mathrm{Cl}$, confidence interval; $\mathrm{AAP}$, abdominal aortic plaque; LDL-C, low density lipoprotein-cholesterol; HDL-C, high density lipoproteincholesterol; CAD, coronary artery disease. 


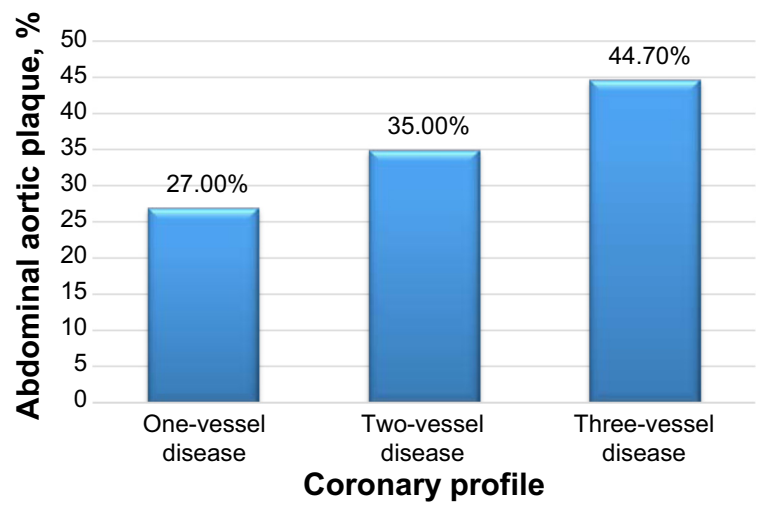

Figure I Prevalence of abdominal aortic plaque according to the coronary profile.

abdominal aortic plaque) were entered into an ordinal logistic regression model. In multivariate analysis, diabetes mellitus, chronic kidney disease, LDL-cholesterol, HDL-cholesterol, and abdominal aortic plaque were the independent factors associated with the severity of CAD (Table 3).

\section{Discussion}

Atherosclerosis is a generalized, progressive disease that may simultaneously affect several arterial trees of the body, such as carotid arteries, femoral arteries, and popliteal arteries., ${ }^{3,17}$ Subclinical atherosclerosis can be noninvasively identified and analyzed to provide prognostic information beyond that provided by traditional cardiovascular risk factors alone as well as to show the cumulative effect of all risk and susceptibility factors. ${ }^{18}$ Due to the increasing trend of cardiovascular and cerebrovascular events in many patient populations, the identification and analysis of subclinical atherosclerosis are of utmost importance for the reduction of these severe events.

Carotid atherosclerosis as shown on ultrasound, aortic atherosclerosis detected by ultrasound, and lower extremity atherosclerosis reflected by the ankle-brachial index are validated measures of atherosclerosis that are routinely used in population-based studies because they are relatively cheap, noninvasive, easy to assess, and cost efficient. ${ }^{18-21}$ Currently, noninvasive imaging modalities for aortic atherosclerotic plaque detection include ultrasound, computed tomography, magnetic resonance imaging, and angiography. ${ }^{1}$

The association between CAD and thoracic aortic plaques has often been reported by using transesophageal echocardiography. ${ }^{6,7}$ Several studies indicated that thoracic plaques may be more strongly associated with CAD than carotid or femoral artery plaques detected by ultrasonography and to be associated with cardiovascular events., ${ }^{9,22}$ Although autopsy study reported plaques in the abdominal aorta, but not in the thoracic aorta, to be severe in patients with cardiac catastrophe, ${ }^{9}$ there are limited studies showing the association between CAD and abdominal aortic plaques. Kazuhiro Ashida et al reported that the prevalence and extents of plaques in both the thoracic and abdominal aortas were greater in patients with CAD than in those without CAD by using CMR. But they reported that plaque in abdominal aorta was not an independent factor for CAD. ${ }^{9-11}$ Although CMR was a useful tool for evaluating atherosclerotic plaques in both the thoracic and abdominal aortas, ${ }^{12}$ it is neither convenient nor cost efficient. In consequence, CMR may not be routinely used in population-based studies for detection of aortic plaques.

Table 3 Factors associated with the severity of CAD (ordinal logistic regression analysis of the I,268 study patients with CAD)

\begin{tabular}{lllll}
\hline Variables & $\begin{array}{l}\text { One-vessel disease } \\
(\mathbf{N}=\mathbf{3 6 3})\end{array}$ & $\begin{array}{l}\text { Two-vessel disease } \\
\mathbf{( N = 3 0 6 )}\end{array}$ & $\begin{array}{l}\text { Three-vessel disease } \\
(\mathbf{N}=\mathbf{5 9 9})\end{array}$ & $\begin{array}{l}\text { Multivariate } \\
\text { analysis }\end{array}$ \\
\hline Age (years) & $63 \pm 10.74$ & $63.24 \pm 10.79$ & $64.09 \pm 10.40$ & 0.445 \\
Sex (male) & $253(69.7)$ & $240(78.4)$ & $454(75.8)$ & 0.814 \\
Smoking & $119(32.8)$ & $124(40.5)$ & $221(36.9)$ & $0.94 I$ \\
Hypertension & $212(58.4)$ & $184(60.1)$ & $382(63.8)$ & 0.789 \\
Diabetes mellitus & $68(18.7)$ & $77(25.2)$ & $220(36.7)$ & $<0.001$ \\
CKD & $52(14.3)$ & $46(15)$ & $135(22.5)$ & 0.027 \\
Triglyceride & $1.54 \pm 1.06$ & $1.75 \pm 1.73$ & $1.63 \pm 1.04$ & 0.778 \\
Total cholesterol & $4.47 \pm 1.23$ & $4.40 \pm 1.29$ & $4.32 \pm 1.22$ & 0.089 \\
LDL-C (mmol/L) & $2.56 \pm 1.04$ & $2.58 \pm 1.03$ & $2.59 \pm 1.03$ & 0.033 \\
HDL-C (mmol/L) & $1.13 \pm 0.03$ & $1.04 \pm 0.26$ & $1.00 \pm 0.24$ & $<0.001$ \\
AAP & $98(27)$ & $107(35)$ & $268(44.7)$ & $<0.001$ \\
\hline
\end{tabular}

Notes: Data are presented as the mean \pm SD or the number (\%) of patients. The dependent variables indicate the severity of CAD according to the number of stenotic coronary vessels. The factors analyzed included age, sex, smoking, hypertension, diabetes mellitus, chronic kidney disease, triglyceride, total cholesterol, LDL-cholesterol, HDL-cholesterol, and abdominal aortic plaque.

Abbreviations: CKD, chronic kidney disease; LDL-C, low density lipoprotein-cholesterol; HDL-C, high density lipoprotein-cholesterol; AAP, abdominal aortic plaque; $\mathrm{CAD}$, coronary artery disease; SD, standard deviation. 
The association between CAD and abdominal aortic plaques has not yet been clarified clearly. Ultrasound imaging has become a useful tool for evaluating atherosclerotic plaques in abdominal aortas, which is relatively cheap, noninvasive, easy to assess, and cost efficient. In the present study, we used ultrasound imaging to determine the prevalence of abdominal aortic plaques and to explore its association with CAD in patients undergoing coronary angiography. The prevalence of abdominal aortic plaques was higher in patients with $\mathrm{CAD}$ than those without CAD. In multivariate analysis, abdominal aortic plaque was an independent factor associated with the presence of CAD. Stepwise increases in the prevalence of abdominal aortic plaque were found to depend on the number of stenotic coronary vessels. Abdominal aortic plaque was an independent factor associated with the severity of CAD.

In our study, we tried to clarify the association between subclinical arterial wall changes in abdominal aortas and the presence and severity of CAD. According to our results, abdominal aortic plaque was found to be an independent factor associated with the presence and severity of CAD. Although atherosclerosis mainly manifests itself in mediumsized vessels, it is also present in the great vessels, such as the abdominal aorta and the carotidartery. ${ }^{15}$ The pathway from risk factors to clinical disease is probably through the development of a subclinical state. Therefore, the identification of these subclinical states may provide a very important marker for the effects of risk factors on the cardiovascular system among relatively asymptomatic individuals. The 2014 ESC guidelines on the diagnosis and treatment of aortic diseases recommended that population screening for AAA should be performed by abdominal aorta ultrasound in all men $>65$ years of age (Class I, Level A). ${ }^{13}$ Our study found that abdominal aorta ultrasound can not only detect AAA but also abdominal aortic plaque, which was an independent factor associated with the presence and severity of CAD. Researchers found that the extent of thoracic aortic atheroma burden was independently associated with increased long-term mortality in patients following cardiothoracic surgery. ${ }^{8}$ This is an attractive hypothesis that this relationship is not limited to patients after surgery, but describes general atherosclerotic disease patterns of the aorta, with impact for prevention of cardiovascular events. Identifying abdominal aortic plaques will be of utmost importance for early diagnosis and intervention of CAD, which will reduce severe cardiovascular events in turn.

\section{Limitations}

There were several limitations in this study. First, our study population was Chinese patients undergoing angiography, who are generally considered to be a highly selected population at high risk for CAD. Because of this selection bias, our results may not be applicable to the general or other ethnic populations. Therefore, screening for abdominal aortic atherosclerosis in an asymptomatic population is necessary. Second, atherosclerosis varies in severity depending on multiple features that contribute to plaque progression and "stability". Plaque instability has been confirmed as a main cause of acute coronary syndrome. ${ }^{23}$ Due to the technical limitations of ultrasound imaging, we cannot assess the stability of abdominal aortic plaque and its association with coronary plaque instability. Recently, researchers have found that targeted fluorescence activatable cell-penetrating peptides (ACPPs) probes distinguished disrupted plaques from stable plaques with high sensitivity and specificity in an animal model. The combination of anatomic, magnetic resonance imaging - derived predictors for disruption and ACPP uptake can further improve the power of identifying high-risk plaques. ${ }^{24}$ With the recent development in technology, it is possible and necessary to further assess the stability of abdominal aortic plaques and their association with CAD. Third, ultrasound imaging was used to evaluate abdominal aortic plaques, but coronary angiography was used to evaluate coronary atherosclerosis, which cannot visualize plaques, and it only shows lumen characteristics. Our current study cannot elucidate the associations of abdominal aortic plaques with coronary artery plaques. Fourth, atherosclerosis is a systemic disease affecting not only the coronary and carotid arteries but also the thoracic and abdominal aorta and the lower extremities. Our present study focused only on abdominal aortic atherosclerosis and CAD.

\section{Conclusion}

In conclusion, the prevalence of abdominal aortic plaques was higher in patients with $\mathrm{CAD}$ than those without CAD. Abdominal aortic plaque was an independent factor found to be associated with the presence and severity of CAD.

\section{Acknowledgments}

This study was supported by the Guangdong Provincial Public Research and Capacity Building Foundation, People's Republic of China (No.2014A020215023) and Science and Technology Program of Guangzhou, People's Republic of China (No.201300000180).

\section{Author contributions}

WL, SL, and JL conceived, designed, and performed the study; analyzed and interpreted data; and drafted the paper. 
YL, WH, and JC designed the study, analyzed and interpreted data, and drafted the paper.

\section{Disclosure}

The authors report no conflicts of interest in this work.

\section{References}

1. Lu Y, Wei J, Shao Q, et al. Assessment of atherosclerotic plaques in the rabbit abdominal aorta with interleukin-8 monoclonal antibody-targeted ultrasound microbubbles. Mol Biol Rep. 2013;40:3083-3092.

2. Barrett-Connor E, Laughlin GA, Connor C. Coronary artery calcium versus intima-media thickness as a measure of cardiovascular disease among asymptomatic adults (from the Rancho Bernardo Study). Am J Cardiol. 2007;99:227-231.

3. Kafetzakis A, Kochiadakis G, Laliotis A, et al. Association of subclinical wall changes of carotid, femoral, and popliteal arteries with obstructive coronary artery disease in patients undergoing coronary angiography. Chest. 2005;128:2538-2543.

4. Belcaro G, Nicolaides AN, Ramaswami G, et al. Carotid and femoral ultrasound morphology screening and cardiovascular events in low risk subjects: a 10-year follow-up study (the CAFES-CAVE study(1)). Atherosclerosis. 2001;156:379-387.

5. Kroger K, Lehmann N, Rappaport L, et al. Carotid and peripheral atherosclerosis in male marathon runners. Med Sci Sports Exerc. 2011;43: $1142-1147$.

6. Agmon Y, Khandheria BK, Meissner I, et al. Relation of coronary artery disease and cerebrovascular disease with atherosclerosis of the thoracic aorta in the general population. Am J Cardiol. 2002;89:262-267.

7. Matsumura Y, Takata J, Yabe T, Furuno T, Chikamori T, Doi YL. Atherosclerotic aortic plaque detected by transesophageal echocardiography: its significance and limitation as a marker for coronary artery disease in the elderly. Chest. 1997;112:81-86.

8. Kurra V, Lieber ML, Sola S, et al. Extent of thoracic aortic atheroma burden and long-term mortality after cardiothoracic surgery: a computed tomography study. JACC Cardiovasc Imaging. 2010;3: $1020-1029$.

9. Ashida K, Momiyama Y, Fayad ZA, et al. Magnetic resonance evaluation of the associations of thoracic and abdominal aortic plaques with the presence and extent of coronary artery stenosis. J Cardiovasc Magn Reson. 2007;9:855-861.

10. Taniguchi H, Momiyama Y, Fayad ZA, et al. In vivo magnetic resonance evaluation of associations between aortic atherosclerosis and both risk factors and coronary artery disease in patients referred for coronary angiography. Am Heart J. 2004;148:137-143.

11. Momiyama Y, Kato R, Fayad ZA, et al. A possible association between coronary plaque instability and complex plaques in abdominal aorta. Arterioscler Thromb Vasc Biol. 2006;26:903-909.
12. Worthley SG, Helft G, Fuster V, et al. High resolution ex vivo magnetic resonance imaging of in situ coronary and aortic atherosclerotic plaque in a porcine model. Atherosclerosis. 2000;150:321-329.

13. Erbel R, Aboyans V, Boileau C, et al. 2014 ESC Guidelines on the diagnosis and treatment of aortic diseases: document covering acute and chronic aortic diseases of the thoracic and abdominal aorta of the adult. The Task Force for the Diagnosis and Treatment of Aortic Diseases of the European Society of Cardiology (ESC). Eur Heart J. 2014;35:2873-2926.

14. Pitsavos CE, Aggeli KI, Barbetseas JD, et al. Effects of pravastatin on thoracic aortic atherosclerosis in patients with heterozygous familial hypercholesterolemia. Am J Cardiol. 1998;82:1484-1488.

15. Kallikazaros IE, Tsioufis CP, Stefanadis CI, Pitsavos CE, Toutouzas PK. Closed relation between carotid and ascending aortic atherosclerosis in cardiac patients. Circulation. 2000;102:III263-III268.

16. Levey AS, Bosch JP, Lewis JB, Greene T, Rogers N, Roth D. A more accurate method to estimate glomerular filtration rate from serum creatinine: a new prediction equation. Modification of Diet in Renal Disease Study Group. Ann Intern Med. 1999;130:461-470.

17. Maranhao RC, Leite AJ. Development of anti-atherosclerosis therapy based on the inflammatory and proliferative aspects of the disease. Curr Pharm Des. 2015;21:1196-204.

18. Sillesen H, Muntendam P, Adourian A, et al. Carotid plaque burden as a measure of subclinical atherosclerosis: comparison with other tests for subclinical arterial disease in the High Risk Plaque Biolmage study. JACC Cardiovasc Imaging. 2012;5:681-689.

19. Sillesen H, Falk E. Why not screen for subclinical atherosclerosis? Lancet. 2011;378:645-646.

20. Erbel R, Mohlenkamp S, Moebus S, et al. Coronary risk stratification, discrimination, and reclassification improvement based on quantification of subclinical coronary atherosclerosis: the Heinz Nixdorf Recall study. J Am Coll Cardiol. 2010;56:1397-1406.

21. van der Meer IM, Bots ML, Hofman A, Del SA, van der Kuip DA, Witteman JC. Predictive value of noninvasive measures of atherosclerosis for incident myocardial infarction: the Rotterdam Study. Circulation. 2004;109:1089-1094.

22. Varga A, Gruber N, Forster T, et al. Atherosclerosis of the descending aorta predicts cardiovascular events: a transesophageal echocardiography study. Cardiovasc Ultrasound. 2004;2:21.

23. Fuster V, Badimon L, Badimon JJ, Chesebro JH. The pathogenesis of coronary artery disease and the acute coronary syndromes (2). N Engl J Med. 1992;326:310-318.

24. Hua N, Baik F, Pham T, et al. Identification of high-risk plaques by MRI and fluorescence imaging in a rabbit model of atherothrombosis. PLoS One. 2015;10:e139833.
Clinical Interventions in Aging

\section{Publish your work in this journal}

Clinical Interventions in Aging is an international, peer-reviewed journal focusing on evidence-based reports on the value or lack thereof of treatments intended to prevent or delay the onset of maladaptive correlates of aging in human beings. This journal is indexed on PubMed Central, MedLine,

\section{Dovepress}

CAS, Scopus and the Elsevier Bibliographic databases. The manuscript management system is completely online and includes a very quick and fair peer-review system, which is all easy to use. Visit http://www.dovepress. com/testimonials.php to read real quotes from published authors. 